\title{
ON CUBIC FOURFOLDS WITH AN INDUCTIVE STRUCTURE
}

\author{
KENJI KOIKE, YAMANASHI UNIVERSITY
}

\begin{abstract}
We study the number of planes for four dimensional projective hypersurfaces which has so-called inductive structure. We also determine transcendental lattices for cubic fourfolds of this type.
\end{abstract}

\section{INTRODUCTION}

Algebraic surfaces in $\mathbb{P}^{3}(\mathbb{C})$ defined by a homogeneous equation

$$
f_{1}(x, y)=f_{2}(z, w)
$$

have been well studied as examples of surfaces with many lines. For example, a smooth quartic surface over $\mathbb{C}$ contains at most 64 lines (Segre, Ram and Schütt, [RS15]) and this bound is realized by Schur's quartic

$$
x\left(x^{3}-y^{3}\right)=z\left(z^{3}-w^{3}\right) .
$$

Let $f(u, v)$ be a Klein's invariant polynomial

$$
f(u, v)=\left(u^{30}+v^{30}\right)+522\left(u^{25} v^{5}-u^{5} v^{25}\right)-10005\left(u^{20} v^{10}+u^{10} v^{20}\right)
$$

for the icosahedral group. Then the surface $f(x, y)=f(z, w)$ of degree 30 has 2700 lines (Boissiére and Sarti, [BS07]). In general, we have

Proposition 1.1 ([BS07, CHM95]). Let $S$ be a smooth surface of degree d defined by (1.1). The number of lines on $S$ is exactly $d\left(d+\alpha_{d}\right)$, where $\alpha_{d}$ is the order of the group of isomorphisms of $\mathbb{P}^{1}(\mathbb{C})$ mapping zeroes of $f_{1}$ to zeroes of $f_{2}$.

In this paper, we study four dimensional hypersurfaces $X$ defined by a homogeneous equation

$$
F_{1}\left(x_{0}, x_{1}, x_{2}\right)=F_{2}\left(y_{0}, y_{1}, y_{2}\right)
$$

with projective coordinates $\left[x_{0}: x_{1}: x_{2}: y_{0}: y_{1}: y_{2}\right] \in \mathbb{P}^{5}(\mathbb{C})$. Note that $X$ is smooth if and only if plane curves

$$
C_{1}: F_{1}\left(x_{0}, x_{1}, x_{2}\right)=y_{0}=y_{1}=y_{2}=0, \quad C_{2}: x_{0}=x_{1}=x_{2}=F_{2}\left(y_{0}, y_{1}, y_{2}\right)=0
$$

are smooth (For simplicity, we will use coordinates $\left[x_{0}: x_{1}: x_{2}\right]$ to represent points on $C_{1}$ ). To state our results, we introduce a few notations and terminologies. We say that a homogeneous polynomial $F\left(x_{0}, x_{1}, x_{2}\right)$ is equivalent to $G\left(x_{0}, x_{1}, x_{2}\right)$ and write $F \sim G$, if there is $g \in \mathrm{GL}_{3}(\mathbb{C})$ such that $F(g \cdot x)=G(x)$. For a homogeneous polynomial $F\left(x_{0}, x_{1}, x_{2}\right)$, we define the group of automorphisms

$$
\operatorname{Aut}(F)=\left\{g \in \mathrm{GL}_{3}(\mathbb{C}) \mid F(g x)=F(x)\right\} .
$$

The group $\operatorname{Aut}(F)$ contains $\mu_{d}=\left\{\zeta \in \mathbb{C} \mid \zeta^{d}=1\right\}$ as scalar matrices, where $d=\operatorname{deg} F$. Next, let $C$ be a plane curve. We call a smooth point $P \in C$ a $k$-flex point if the tangent line at $P$ and $C$ intersect with intersection multiplicity $k \geq 3$. We will show the following Proposition in Section 2.

Proposition 1.2. Let $X \subset \mathbb{P}^{5}(\mathbb{C})$ be a smooth hypersurface of degree $d \geq 3$ defined by (1.2), and $\nu_{i}$ be the number of $d$-flex points.

(1) If $F_{1} \sim F_{2}$, the number of planes in $X$ is $d \nu_{1}^{2}+\left|\operatorname{Aut}\left(F_{1}\right)\right|$.

(2) If $F_{1} \nsim F_{2}$, the number of planes in $X$ is $d \nu_{1} \nu_{2}$.

In the cases of $d=3$, we have 
Corollary 1.3. Let $X$ be a smooth cubic fourfold defined by the equation (1.2).

(1) If $F_{1} \sim F_{2}$, the number of planes in $X$ is $\left\{\begin{array}{l}243+162 \quad(j=0) \\ 243+108 \quad(j=1728) \\ 243+54 \quad(j \neq 0,1728)\end{array}\right.$, where $j$ is the $j$-invariant of $C_{i}$ as an elliptic curve.

(2) If $F_{1} \nsim F_{2}$, then $X$ contains 243 planes.

Note that $X$ is isomorphic to the Fermat cubic fourfold if $j=0$. Recently, there is a progress in the number of planes in cubic fourfolds by Degtyarev, Itenberg and J. C. Ottem.

Theorem 1.4 ([DIO21]). Let $X \subset \mathbb{P}^{5}(\mathbb{C})$ be a smooth cubic fourfold. Then, either $X$ has at most 350 planes, or, up to projective equivalence, $X$ is

(1) the Fermat cubic (with 405 planes), or

(2) the Clebsch Segre cubic (with 357 planes), or

(3) the cubic fourfold with 351 planes.

The Approach in [DIO21] is based on the lattice theorey and the Torelli theorem, and it seems that the cubic fourfold with 351 planes is not given explicitly. It is just the second case of (1) in Corollary 1.3 .

The second purpose of this paper is to determine transcendental lattices for cubic fourfolds of this type. The middle cohomology for hypersurfaces of this type was studied by Shioda and Katsura using "inductive structure" ([SK79], [Sh79]). Let us consider smooth algebraic suraces of degree $d$

$$
S_{1}: F_{1}\left(x_{0}, x_{1}, x_{2}\right)=x_{3}^{d}, \quad S_{1}: F_{2}\left(y_{0}, y_{1}, y_{2}\right)=y_{3}^{d}
$$

that are cyclic coverings of $\mathbb{P}^{2}$ branched along $C_{1}$ and $C_{2}$ respectively. We have a dominant rational map $S_{1} \times S_{2} \rightarrow X$ defined by

$$
\left[x_{0}: \cdots: x_{3}\right] \times\left[y_{0}: \cdots: y_{3}\right] \mapsto\left[y_{3} x_{0}: y_{3} x_{1}: y_{3} x_{2}: x_{3} y_{0} x_{3} y_{1}: x_{3} y_{2}\right] .
$$

Theorem 1.5 ([SK79], Sh79]). This rational map induces an isomorphism

$$
\left[H_{\text {prim }}^{2}\left(S_{1}, \mathbb{C}\right) \otimes H_{\text {prim }}^{2}\left(S_{2}, \mathbb{C}\right)\right]^{\mu_{d}} \oplus\left[H^{1}\left(C_{1}, \mathbb{C}\right) \otimes H^{1}\left(C_{2}, \mathbb{C}\right)\right](1) \stackrel{\sim}{\rightarrow} H_{\text {prim }}^{4}(X, \mathbb{C})
$$

where $\mu_{d}$ acts as

$$
\left[x_{0}: y_{1}: x_{2}: x_{3}\right] \times\left[y_{0}: y_{1}: y_{2}: y_{3}\right] \mapsto\left[x_{0}: y_{1}: x_{2}: \zeta x_{3}\right] \times\left[y_{0}: y_{1}: y_{2}: \zeta y_{3}\right] .
$$

Namely, the Hodge structure of $X$ is determined by that of $C_{i}$ and $S_{i}$. In a different context, Beauville noted

Proposition 1.6 ([Be14]). Let $F$ be a cubic form in 3 variables, such that the curve $F(x, y, z)=$ 0 in $\mathbb{P}^{2}$ is an elliptic curve with complex multiplication. Let $X$ be the cubic fourfold defined by $F\left(x_{0}, x_{1}, x_{2}\right)=F\left(y_{0}, y_{1}, y_{2}\right)$ in $\mathbb{P}^{5}$. Then $H^{4}(X, \mathbb{Z})_{\text {alg }}$ has maximal rank $h^{2,2}(X)$.

Computing intersection numbers of transcendental cycles coming from $C_{1} \times C_{2}$, we determine the transcwndemtal lattice for cubic fourfolds of this type. Note that the integral Hodge conjecture is known for cubic fourfolds (V07]). In Section 3 and 4, we show

Theorem 1.7. Let $X$ be a smooth cubic fourfold defined by the equation (1.2). We have a morphism of integral Hodge structures

$$
\phi:\left[H^{1}\left(C_{1}, \mathbb{Z}\right) \otimes H^{1}\left(C_{2}, \mathbb{Z}\right)\right](1) \longrightarrow H^{4}(X, \mathbb{Z})
$$

such that $\phi(\alpha) \cdot \phi(\beta)=-3(\alpha \cdot \beta)$. For a general $X$, the transcendental lattice $T_{X}$ of $X$, that is, the orthogonal complement of $H^{2,2}(X) \cap H^{4}(X, \mathbb{Z})$ is given by

$$
T_{X}=\operatorname{Im} \phi=\mathrm{U}(3) \oplus \mathrm{U}(3) .
$$


Let us recall the following formula by Shioda and Mitani ([SM74]). For a positive definite even latiice $T=\left[\begin{array}{cc}2 a & b \\ b & 2 c\end{array}\right](a, b, c \in \mathbb{Z})$ of rank 2, we have an Abelian surface $C_{1} \times C_{2}$ whose transcendental lattice is $T$, where $C_{i}=\mathbb{C} /\left(\mathbb{Z}+\mathbb{Z} \tau_{i}\right)$ and

$$
\tau_{1}=\frac{-b+\sqrt{\Delta}}{2 a}, \quad \tau_{2}=\frac{b+\sqrt{\Delta}}{2} \quad\left(\Delta=b^{2}-4 a c\right)
$$

Corollary 1.8. Let $F_{i}\left(x_{0}, x_{1}, x_{2}\right)=0$ be the cubic equation of the above elliptic curve $C_{i}$. Then the corresponding cubic fourfold $X$ defined by $F_{1}(x)=F_{2}(y)$ has the transcendental lattice $T(-3)=\left[\begin{array}{ll}-6 a & -3 b \\ -3 b & -6 c\end{array}\right]$.

For example, we have $\tau_{1}=\tau_{2}=\sqrt{-1}$ for $a=c=1, b=0$. The corresponding cubic fourfold $X$ (the case of $j=1728$ in Corollary [1.3) has the transcendental lattice $\left[\begin{array}{cc}-6 & 0 \\ 0 & -6\end{array}\right]$, which was computed as the transcendental lattice for the cubic fourfold with 351 planes in [DIO21].

\section{NUMBER OF PLANES}

Let $S$ be a plane in $\mathbb{P}^{5}(\mathbb{C})$ defined by a system of linear equations

$$
\left\{\begin{array}{l}
a_{00} x_{0}+a_{01} x_{1}+a_{02} x_{2}=b_{00} y_{0}+b_{01} y_{1}+b_{02} y_{2} \\
a_{10} x_{0}+a_{11} x_{1}+a_{12} x_{2}=b_{10} y_{0}+b_{11} y_{1}+b_{12} y_{2} \\
a_{20} x_{0}+a_{21} x_{1}+a_{22} x_{2}=b_{20} y_{0}+b_{21} y_{1}+b_{22} y_{2}
\end{array}\right.
$$

of rank 3, which is denoted by $A x=B y$ where $A=\left[a_{i j}\right]$ and $B=\left[b_{i j}\right]$. Let $X$ be a smooth hypersurface in $\mathbb{P}^{5}(\mathbb{C})$ defined by (1.2) .

Lemma 2.1. We assume that $\operatorname{deg} X \geq 2$ and $S \subset X$. Then, we have

(1) $\operatorname{rank} A, \operatorname{rank} B \geq 2$,

(2) $\operatorname{rank} A=3 \Leftrightarrow \operatorname{rank} B=3 \quad \Rightarrow \quad F_{1} \sim F_{2}$,

(3) $\operatorname{rank} A=2 \Leftrightarrow \operatorname{rank} B=2$.

Proof. (1) If $\operatorname{rank} B=0$, then we have $b_{i, j}=0$ for all $i, j$ and the system (2.1) is equivalent to $x_{0}=x_{1}=x_{2}=0$. By the assumption $S \subset X$, we have

$$
F_{1}(0,0,0)=F_{2}\left(y_{0}, y_{2}, y_{3}\right)
$$

and this is not the case. Let us assume that $\operatorname{rank} B=1$. Then, the system (2.1) is reduced to

$$
\begin{aligned}
& \ell_{0}\left(x_{0}, x_{1}, x_{2}\right)=m_{0}\left(y_{0}, y_{1}, y_{2}\right) \\
& \ell_{1}\left(x_{0}, x_{1}, x_{2}\right)=0 \\
& \ell_{2}\left(x_{0}, x_{1}, x_{2}\right)=0
\end{aligned}
$$

where $\ell_{i}$ and $m_{0}$ are linear forms. By the assumption $S \subset X$, there exist homogeneous polynomials $q_{i}$ of degree $d-1$ such that

$$
F_{1}(x)-F_{2}(y)=q_{0}(x, y)\left(\ell_{0}(x)-m_{0}(y)\right)+q_{1}(x, y) \ell_{1}(x)+q_{2}(x, y) \ell_{2}(x)
$$

Putting $x_{0}=x_{1}=x_{2}=0$, we have $F_{2}(y)=q_{0}(0, y) m_{0}(y)$ and this contradicts irreducibility of $F_{2}$. As above, we have $\operatorname{rank} B \geq 2$ and the same is true for $A$.

(2) Let us assume that $\operatorname{rank} B=3$. The plane $S$ is given by $B^{-1} A x=y$ and we have an identity $F_{1}(x)=F_{2}\left(B^{-1} A x\right)$ as a polynomial of $x_{0}, x_{1}, x_{2}$. Since $X$ is smooth, $F_{1}(x)=0$ is a smooth plane curve and so is $F_{2}\left(B^{-1} A x\right)=0$. We see that $B^{-1} A \in \mathrm{GL}_{3}(\mathbb{C})$ (note that $\operatorname{deg} X \neq 1)$ and $F_{1} \sim F_{2}$. The same is true for the case rank $A=3$.

(3) By (1) and (2), we see that $\operatorname{rank} B=2$ if and only if $\operatorname{rank} A=2$.

In the following, we say that a plane $S$ is of $\operatorname{rank} k$ if $\operatorname{rank} A=k$.

Lemma 2.2. If $F_{1} \sim F_{2}$, there exist exactly $\left|\operatorname{Aut}\left(F_{1}\right)\right|$ planes of rank 3 in $X$. 
Proof. We may assume that $F_{1}=F_{2}$. If $A x=B y$ is a plane of rank 3 in $X$, we have $F_{1}(x)=F_{1}\left(B^{-1} A x\right)$ and $B^{-1} A \in \operatorname{Aut}\left(F_{1}\right)$. Conversely, $y=g x$ is a plane of rank 3 in $X$ for any $g \in \operatorname{Aut}\left(F_{1}\right)$.

Before we consider the number of planes of rank 2, we note that

Lemma 2.3. Let $\phi_{1}\left(x_{0}, x_{1}\right)$ and $\phi_{2}\left(y_{0}, y_{1}\right)$ be homogeneous polynomials of degree $d$. If $\phi_{1}-\phi_{2}$ is devided by $x_{1}-y_{1}$, then $\phi_{1}-\phi_{2}$ is a scalar multiple of $x_{1}^{d}-y_{1}^{d}$.

Proof. We have

$$
\phi_{1}-\phi_{2} \equiv 0 \quad \bmod x_{1}-y_{1} \quad \Leftrightarrow \quad \phi_{1}\left(x_{0}, x_{1}\right)-\phi_{2}\left(y_{0}, x_{1}\right)=0 \quad \text { in } \mathbb{C}\left[x_{0}, x_{1}, y_{0}\right],
$$

and a binary forms $\phi_{1}\left(x_{0}, x_{1}\right)$ coincide with $\phi_{2}\left(y_{0}, x_{1}\right)$ if and only if

$$
\phi_{1}\left(x_{0}, x_{1}\right)=\phi_{2}\left(y_{0}, x_{1}\right)=\text { const. } \times x_{1}^{d} .
$$

Now let us assume that $\operatorname{rank} A=\operatorname{rank} B=2$. Then the equation of $S$ is reduced to the following form

$$
\begin{aligned}
\ell_{0}\left(x_{0}, x_{1}, x_{2}\right) & =0 \\
\ell_{1}\left(x_{0}, x_{1}, x_{2}\right) & =m_{1}\left(y_{0}, y_{1}, y_{2}\right) \\
0 & =m_{2}\left(y_{0}, y_{1}, y_{2}\right)
\end{aligned}
$$

where $\ell_{i}$ and $m_{i}$ are linear forms. Note that $\ell_{0}(x)$ and $m_{2}(y)$ are uniquely determined by $S$, up to scalr multiplication.

Lemma 2.4. We assume that $d=\operatorname{deg} X \geq 2$ and $S \subset X$.

(1) If $S$ is given by the above form, $\ell_{0}(x)=0$ is a d-flex tangent of $C_{1}$ (we regard $\left[x_{0}: x_{1}: x_{2}\right]$ as coordinates of $\mathbb{P}^{2}(\mathbb{C})$ ). The same is true for $m_{2}(y)=0$ and $C_{2}$.

(2) Conversely, a pair of d-flex tangents of $C_{1}$ and $C_{2}$ gives $d$ planes of rank 2 in $X$.

(3) There exist exactly $d \nu_{1} \nu_{2}$ planes of rank 2 in $X$, where $\nu_{i}$ is the number of $d$-flex points of $C_{i}$.

Proof. (1) For simplicity, we take projectve coodinates as

$$
\ell_{0}\left(x_{0}, x_{1}, x_{2}\right)=x_{2}, \quad \ell_{1}\left(x_{0}, x_{1}, x_{2}\right)=x_{1}, \quad m_{1}\left(y_{0}, y_{1}, y_{2}\right)=y_{1}, \quad m_{2}\left(y_{0}, y_{1}, y_{2}\right)=y_{2}
$$

(this is possible by the action of $\mathrm{GL}_{3}(\mathbb{C}) \times \mathrm{GL}_{3}(\mathbb{C})$, since $\operatorname{rank} A=\operatorname{rank} B=2$ ). Namely, we assume that $S$ is given by

$$
x_{2}=0, \quad x_{1}-y_{1}=0, \quad y_{2}=0 .
$$

By the assumption $S \subset X$, we see that $F_{1}\left(x_{0}, x_{1}, 0\right)-F_{2}\left(y_{0}, y_{1}, 0\right)$ is divided by $x_{1}-y_{1}$. By lemma 2.3, we have

$$
F_{1}\left(x_{0}, x_{1}, 0\right)=c x_{1}^{d}, \quad F_{2}\left(y_{0}, y_{1}, 0\right)=c y_{1}^{d}
$$

where $c$ is a constant. This implies that $x_{2}=0$ is a $d$-flex tangent of $C_{1}$ and $y_{2}=0$ is a $d$-flex tangent of $C_{2}$.

(2) Let $\ell_{0}(x)=0$ (resp. $m_{2}(y)=0$ ) be a $d$-flex tangent of $C_{1}$ (resp. $C_{2}$ ). There exist homogeneous polynomials $G_{1}, G_{2}$ of degree $d-1$ and linear forms $\ell_{1}, m_{1}$ such that

$$
F_{1}(x)=\ell_{0}(x) G_{1}(x)+\ell_{1}(x)^{d}, \quad F_{2}(y)=m_{2}(y) G_{2}(y)+m_{1}(y)^{d} .
$$

Since $F_{1}$ is irreducible, $\ell_{0}$ is not a scalar multiple of $\ell_{1}$. The same is true for $m_{1}$ and $m_{2}$. We see that a system of linear equations

$$
\begin{aligned}
\ell_{0}\left(x_{0}, x_{1}, x_{2}\right) & =0 \\
\ell_{1}\left(x_{0}, x_{1}, x_{2}\right) & =\zeta m_{1}\left(y_{0}, y_{1}, y_{2}\right) \\
0 & =m_{2}\left(y_{0}, y_{1}, y_{2}\right)
\end{aligned}
$$

gives a plane in $X$ for any $\zeta \in \mu_{d}$.

(3) By the above construction, we see that the number of planes of rank 2 in $X$ is $d \nu_{1} \nu_{2}$. 
Proposition 1.2 follows from Lemma 2.1, 2.2 and 2.4. In the last of this section, we consider the case of $d=3$. It is well known that a smooth cubic curve has nine inflection points. Hence a smooth cubic 4 -fold $X$ of this type contains $3 \times 9 \times 9=243$ planes of rank 2 . In the case of $F_{1} \sim F_{2}$, we have extra $\left|\operatorname{Aut}\left(F_{1}\right)\right|$ planes of rank 3. To see automorphisms of ternary cubic forms, the Hesse normal form

$$
F\left(x_{0}, x_{1}, x_{2}\right)=x_{0}^{3}+x_{1}^{3}+x_{2}^{3}-3 \lambda x_{0} x_{1} x_{2}
$$

is usefull (see, e.g. [BM17]). It defines a smooth cubic curve if $\lambda \notin \mu_{3}$, and we have the j-invariant

$$
j=1728 \frac{\lambda^{3}\left(\lambda^{3}+8\right)^{3}}{64\left(\lambda^{3}-1\right)^{3}}=1728 \frac{g_{2}^{3}}{g_{2}^{3}-27 g_{3}^{2}} .
$$

In general, the group $\operatorname{Aut}(F) / \mu_{3} \subset \mathrm{PGL}_{3}(\mathbb{C})$ is of order 18 generated by permutations of coordinates and an automorphism

$$
\left(x_{0}, x_{1}, x_{2}\right) \mapsto\left(x_{0}, \omega x_{1}, \omega^{2} x_{2}\right)
$$

where $\omega=e^{2 \pi i / 3}$ (If we take a flex point as the orgin $O$, this is the group generated by the inversion and translations by 3 -tosions). As is well known, we have extra automorphisms for $j=0,1728$. For example, if $\lambda=0$ (and hence $j=0$ ), we have an automorphism

$$
\left(x_{0}, x_{1}, x_{2}\right) \mapsto\left(x_{0}, x_{1}, \omega x_{2}\right)
$$

of order 3. As an example for $j=1728$, if $\lambda=1+\sqrt{3}$, we have an automorphism

$$
M=-\frac{1}{\sqrt{3}}\left[\begin{array}{ccc}
1 & 1 & 1 \\
1 & \omega & \omega^{2} \\
1 & \omega^{2} & \omega
\end{array}\right] \in \mathrm{GL}_{3}(\mathbb{C})
$$

of order 4 .

Remark 2.5. For the Hesse normal form, we have the following flex points and tangent lines.

$$
\begin{array}{c|c||c|c}
{[0:-1: 1]} & \lambda x_{0}+x_{1}+x_{2}=0 \\
{[0:-\omega: 1]} & \omega \lambda x_{0}+\omega^{2} x_{1}+x_{2}=0 & {\left[1: 0:-\omega^{2}\right]} & x_{0}+\lambda w^{2} x_{1}+\omega x_{2}=0 \\
{\left[0:-\omega^{2}: 1\right]} & \omega^{2} \lambda x_{0}+\omega x_{1}+x_{2}=0 & {\left[\begin{array}{c}
1: 0 \\
0
\end{array}\right.} & x_{0}+x_{1}+\lambda x_{2}=0 \\
{[1: 0:-1]} & x_{0}+\lambda x_{1}+x_{2}=0 \\
{[1: 0:-\omega]} & x_{0}+\lambda \omega x_{1}+\omega^{2} x_{2}=0 & \omega^{2} x_{0}+x_{1}+\lambda \omega x_{2}=0 \\
{\left[-\omega^{2}: 1: 0\right]} & \omega x_{0}+x_{1}+\lambda \omega^{2} x_{2}=0
\end{array}
$$

\section{TRANSCENDENTAL CYCLES}

3.1. transcendental lattice. We compute intersection numbers of topological 4-cycles in $X$ coming from $C_{1} \times C_{2}$. Let us consider a divisor

$$
V: F_{1}(x)=F_{2}(y)=0
$$

of $X$. It is singular along $C_{1} \cup C_{2}$, and the projection

$$
p: V-C_{1} \cup C_{2} \longrightarrow C_{1} \times C_{2}, \quad[x: y] \mapsto\left[x_{0}: x_{1}: x_{2}\right] \times\left[y_{0}: y_{1}: y_{2}\right]
$$

gives a structure of $\mathbb{C}^{*}$-bundle. Let $\pi: \widetilde{X} \rightarrow X$ be the blow up of $X$ along $C_{1} \cup C_{2}$ and $\widetilde{V}$ be the strict transform of $V$. Then we have a $\mathbb{P}^{1}$-bundle $p: \widetilde{V} \rightarrow C_{1} \times C_{1}$. 


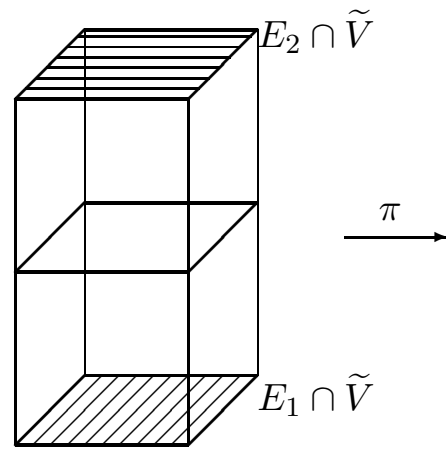

$\widetilde{V}$

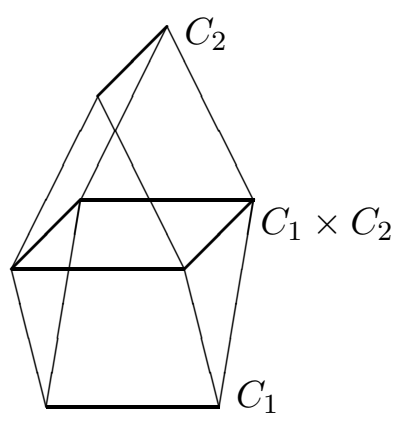

$V$

Let $\iota: \widetilde{V} \rightarrow \widetilde{X}$ be the inclusion map. We have the following diagram

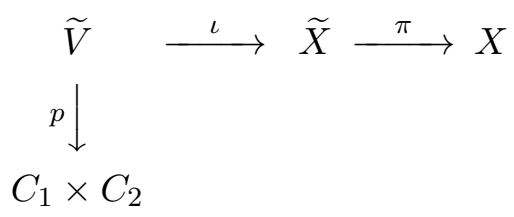

and a morphism of integral Hodge structures

$$
(\pi \circ \iota)_{*} \circ p^{*}: H^{2}\left(C_{1} \times C_{2}, \mathbb{Z}\right)(1) \longrightarrow H^{4}(X, \mathbb{Z})
$$

where the pushforward map $(\pi \circ \iota)_{*}$ is defined via the Poincaré duality (In the following, we often identify cohomology groups and homology groups). Our interset is the restriction on a Künneth component

$$
\phi:\left[H^{1}\left(C_{1}, \mathbb{Z}\right) \otimes H^{1}\left(C_{2}, \mathbb{Z}\right)\right](1) \longrightarrow H^{4}(X, \mathbb{Z}) .
$$

Lemma 3.1. Let $\gamma_{i}$ be a topological 1-cycle on $C_{i}$ and put

$$
\Gamma=p^{-1}\left(\gamma_{1} \times \gamma_{2}\right) \subset \widetilde{V} \subset \widetilde{X} .
$$

For $[\Gamma] \in H^{4}(\tilde{X}, \mathbb{Z})$, we have $\pi^{*} \pi_{*}[\Gamma]=[\Gamma]$.

Proof. It is enough to show in the case that $\gamma_{1}$ and $\gamma_{2}$ are smooth and isomorphic to a circle $S^{1}$ since general 1-cycles are homologous to $\mathbb{Z}$-linear combinations of them. Namely, we assume that $\Gamma$ is a $\mathbb{P}^{1}(\mathbb{C})$-bundle over $S^{1} \times S^{1}$.

Let $E_{i}=\pi^{-1}\left(C_{i}\right)$ be the exceptional divisor. First we show that $\Gamma$ and $E_{i}$ intersect transversally in $\widetilde{X}$. Since $C_{1} \cap C_{2}=\phi$ and the problem is local, we consider the blow up along only $C_{1}$. Then $\widetilde{X}$ is realized in $\mathbb{P}^{5}(\mathbb{C}) \times \mathbb{P}^{2}(\mathbb{C})$ as

$$
F_{1}\left(x_{0}, x_{1}, x_{2}\right)=F_{2}\left(y_{0}, y_{1}, y_{2}\right), \quad \operatorname{rank}\left[\begin{array}{ccc}
y_{0} & y_{1} & y_{2} \\
t_{0} & t_{1} & t_{2}
\end{array}\right]=1
$$

where $\left[t_{0}: t_{1}: t_{2}\right] \in \mathbb{P}^{2}(\mathbb{C})$. In an affine open set given by $x_{0}=1$ and $t_{0}=1$ (that is $\left.y_{1}=t_{1} y_{0}, y_{2}=t_{2} y_{0}\right)$, we have

$$
\begin{aligned}
\widetilde{X} & : F_{1}\left(1, x_{1}, x_{2}\right)=y_{0}^{d} F_{2}\left(1, t_{1}, t_{2}\right) \\
\widetilde{V} & : F_{1}\left(1, x_{1}, x_{2}\right)=F_{2}\left(1, t_{1}, t_{2}\right)=0 \\
E_{1}: F_{1}\left(1, x_{1}, x_{2}\right) & =y_{0}=0
\end{aligned}
$$

where $\left(x_{1}, x_{2}, y_{0}, t_{1}, t_{2}\right) \in \mathbb{C}^{5}$. The exceptional divisor $E_{1}$ is given by $y_{0}=0$ in $\tilde{X}$, and $y_{0}$ is a (complex) local coordinate for the fiber direction of $\widetilde{V}$. We have same situations in other affine charts. From this, we see transversality of $E_{1}$ and $\Gamma$, that is, $T_{x} E_{1}+T_{x} \Gamma=T_{x} \widetilde{X}$ for $x \in E_{1} \cap \Gamma$. 
Now we have $E_{1} \cap \Gamma \cong \gamma_{1} \times \gamma_{2}=S^{1} \times S^{1}$ (a smooth section of a fiber bundle $\Gamma \rightarrow S^{1} \times S^{1}$ ), and

$$
i_{1}^{*}[\Gamma]=\left[\Gamma \cap E_{1}\right] \in H_{2}\left(E_{1}, \mathbb{Z}\right) \cong H^{4}\left(E_{1}, \mathbb{Z}\right)
$$

where $i_{1}: E_{1} \rightarrow \widetilde{X}$ is the inclusion map. Note that the projection $\pi$ gives fibrations

$$
\begin{array}{cccc}
E_{1} & \longrightarrow & C_{1} & \mathbb{P}^{2}(\mathbb{C}) \text {-bundle } \\
\cup & & \cup & \\
\left(\Gamma \cap E_{1}\right) & \longrightarrow & \gamma_{1}=S^{1} & \gamma_{2} \text {-bundle }\left(S^{1} \text {-bundle }\right)
\end{array}
$$

and $\Gamma \cap E_{1}$ is the boudary of a solid torus in $E_{1}$. Therefore we have $i_{1}^{*}[\Gamma]=0$, and the same is true for the pull-back to $H_{2}\left(E_{2}, \mathbb{Z}\right)$. This implies that $[\Gamma]=\pi^{*} \alpha$ for some $\alpha \in H^{4}(X, \mathbb{Z})$ since there is an exact sequence

$$
H^{4}(X, \mathbb{Z}) \longrightarrow H^{4}(\tilde{X}, \mathbb{Z}) \longrightarrow H^{4}(E, \mathbb{Z}) .
$$

Therefore we have $\pi^{*} \pi_{*}[\Gamma]=\pi^{*} \pi_{*} \pi^{*} \alpha=\pi^{*} \alpha=[\Gamma]$.

Lemma 3.2. (1) The morphism $\phi$ in (3.2) satisfies

$$
\phi(\alpha) \cdot \phi(\beta)=-(\operatorname{deg} X)(\alpha \cdot \beta) .
$$

In particular, the image $\operatorname{Im} \phi$ is a sublattice of rank $4 g^{2}$ with the intersection form

$$
\left[\begin{array}{ll}
0 & d \\
d & 0
\end{array}\right] \oplus \cdots \oplus\left[\begin{array}{ll}
0 & d \\
d & 0
\end{array}\right]\left(2 g^{2} \text { times }\right)
$$

where $d=\operatorname{deg} X$ and $g=\frac{(d-1)(d-2)}{2}$ is the genus of $C_{i}$.

(2) For a plane $S \subset X$ of rank 2 , the class $[S] \in H^{4}(X, \mathbb{Z})$ is orthogonal to $\operatorname{Im} \phi$.

Proof. (1) For $\alpha, \beta \in H^{1}\left(C_{1}, \mathbb{Z}\right) \otimes H^{1}\left(C_{2}, \mathbb{Z}\right)$, we denote $p^{*} \alpha$ and $p^{*} \beta$ by $A$ and $B$. By the projection formula and the previous Lemma, we have

$$
\begin{aligned}
\phi(\alpha) \cdot \phi(\beta) & =\pi_{*}\left(\iota_{*} A\right) \cdot \pi_{*}\left(\iota_{*} B\right) \\
& =\pi_{*}\left(\left(\pi^{*} \pi_{*} \iota_{*} A\right) \cdot \iota_{*} B\right)=\pi_{*}\left(\iota_{*} A \cdot \iota_{*} B\right) .
\end{aligned}
$$

We can compute this excess intersection by

$$
\left(\iota_{*} A \cdot \iota_{*} B\right)_{\widetilde{X}}=\left(A \cdot B \cdot \iota^{*}[\widetilde{V}]\right)_{\widetilde{V}}=\left(p^{*} \alpha \cdot p^{*} \beta \cdot \iota^{*}[\widetilde{V}]\right)_{\widetilde{V}}
$$

where $(-\cdot-)_{M}$ represents the intersection product in $M$, and $[\widetilde{V}] \in H^{2}(\widetilde{X}, \mathbb{Z})$ is the class of $\widetilde{V}$. Let $F \cong \mathbb{P}^{1}(\mathbb{C})$ be a fiber of $\widetilde{V}$. Then we have

$$
p^{*} \alpha \cdot p^{*} \beta=(\alpha \cdot \beta)_{C_{1} \times C_{2}} \cdot[F]
$$

and hence

$$
\phi(\alpha) \cdot \phi(\beta)=(\alpha \cdot \beta) \cdot\left([F] \cdot \iota^{*}[\tilde{V}]\right) .
$$

Lastly, let $[H] \in H^{2}(X, \mathbb{Z})$ be the hyperplane class and $E_{i}$ be exceptinal divisors $\pi^{-1}\left(C_{i}\right)$. We have

$$
[\widetilde{V}]=\pi^{*}[V]-d\left[E_{1}\right]-d\left[E_{2}\right]=d\left(\pi^{*}[H]-\left[E_{1}\right]-\left[E_{2}\right]\right) .
$$

By calculation with local coordinates in the previous Lemma, we see that

$$
[F] \cdot \pi^{*}[H]=1, \quad[F] \cdot \pi^{*}\left[E_{i}\right]=1 .
$$

Therefore we have $[F] \cdot \iota^{*}[\widetilde{V}]=-d$, and we conclude the formula. Since the intersection form on $H^{1}\left(C_{1}, \mathbb{Z}\right) \otimes H^{1}\left(C_{2}, \mathbb{Z}\right) \cong \mathbb{Z}^{4 g^{2}}$ is

$$
\left[\begin{array}{ll}
0 & 1 \\
1 & 0
\end{array}\right] \oplus \cdots \oplus\left[\begin{array}{ll}
0 & 1 \\
1 & 0
\end{array}\right]\left(2 g^{2} \text { times }\right)
$$


we complete the proof of (1).

(2) We may assume that a topological 1-cycle $\gamma_{i}$ on $C_{i}$ does not pass through any $d$-flex points on $C_{i}$, and that a plane $S \subset X$ of rank 2 is disjoint from $\operatorname{Im} \phi$.

\section{CUBIC 4-FOLDS}

4.1. cubic 4-folds. Let $X \subset \mathbb{P}^{5}(\mathbb{C})$ be a smooth cubic hypersurface. We have

$$
h^{4,0}(X)=0, \quad h^{3,1}(X)=1, \quad h^{2,2}(X)=21
$$

and the integral middle cohomology $H^{4}(X, \mathbb{Z})$ is the unimodular lattice $(+1)^{\oplus 21} \oplus(-1)^{\oplus 2}$. The integral Hodge conjecture is known to be valid, that is, $H^{2,2}(X) \cap H^{4}(X, \mathbb{Z})$ is spanned by algebraic cycles $([\mathrm{V} 07])$. Let $T_{X} \subset H^{4}(X, \mathbb{Z})$ be the orthogonal complement of algebraic cycles. We call it the transcendental lattice of $X$. In the following, we show that $T_{X}$ for a general cubic fourfold $X$ given by $F(X)=F(y)$ is

$$
\operatorname{Im} \phi=\left[\begin{array}{ll}
0 & 3 \\
3 & 0
\end{array}\right] \oplus\left[\begin{array}{ll}
0 & 3 \\
3 & 0
\end{array}\right] .
$$

4.2. Fermat cubic 4-fold. In [DS16], Degtyarev and Shimada studied the sublattice of the middle homology group of Fermat varieties generated by the classes of linear subspaces. They gave an algebraic (or rather combinatorial) criterion for the primitivity of this submodule. To apply this result, we take

$$
F_{1}=z_{0}^{3}+z_{1}^{3}+z_{2}^{3}, \quad F_{2}=-\left(z_{3}^{3}+z_{4}^{3}+z_{5}^{3}\right) .
$$

Namely, we consider the Fermat cubic 4-fold

$$
X_{0}: F_{1}-F_{2}=\left(z_{0}^{3}+z_{1}^{3}+z_{2}^{3}\right)+\left(z_{3}^{3}+z_{4}^{3}+z_{5}^{3}\right)=0 .
$$

According to Degtyarev and Shimada, let $\mathcal{K}$ be the set of indices

$$
J_{1}=[01|23| 45], \quad J_{2}=[01|24| 35], \quad J_{3}=[02|13| 45], \quad J_{4}=[02|14| 35] .
$$

For $J=\left[j_{0}, k_{0}\left|j_{1}, k_{1}\right| j_{2}, k_{2}\right] \in \mathcal{K}$ and $\beta=\left(\beta_{0}, \beta_{1}, \beta_{2}\right) \in \mathcal{B}=\left(\mu_{3}\right)^{3}$, we define a plane

$$
L_{J, \beta}: z_{k_{0}}=-\beta_{0} z_{j_{0}}, \quad z_{k_{1}}=-\beta_{1} z_{j_{1}}, \quad z_{k_{2}}=-\beta_{0} z_{j_{2}}
$$

in $X$. Note that these planes are of rank 2, that is, induced by flex tangents of $C_{i}$. Let $\mathcal{L}_{\mathcal{K}}$ be the submodule of $H^{4}\left(X_{0}, \mathbb{Z}\right)$ generated by $\left[L_{J, \beta}\right]$. By Theorem 1.1 in [DS16], the torsions of the quotient module $H^{4}\left(X_{0}, \mathbb{Z}\right) / \mathcal{L}_{\mathcal{K}}$ is isomorphic to the torsions of

$$
\mathbb{Z}\left[t_{1}, t_{2}, t_{3}, t_{4}, t_{5}\right] /\left(t_{i}^{2}+t_{i}+1, \rho_{J} \mid i=1, \ldots, 5, J \in \mathcal{K}\right),
$$

where

$$
\rho_{J}=\left(1+t_{j_{1}}+t_{j_{1}} t_{k_{1}}\right)\left(1+t_{j_{2}}+t_{j_{2}} t_{k_{2}}\right) .
$$

Lemma 4.1. The module (4.1) is torsion-free. Therefore the sublattice $\mathcal{L}_{\mathcal{K}}$ is primitive in $H^{4}\left(X_{0}, \mathbb{Z}\right)$.

Proof. Note that the quotient

$$
\mathbb{Z}\left[t_{1}, t_{2}, t_{3}, t_{4}, t_{5}\right] /\left(t_{i}^{2}+t_{i}+1 \mid i=1, \ldots, 5\right)
$$

is a free module of rank $2^{5}$, generated by monomials $t^{q}=t_{1}^{q_{1}} t_{2}^{q_{2}} t_{3}^{q_{3}} t_{4}^{q_{4}} t_{5}^{q}$ with $q_{i} \in\{0,1\}$. On the other hand, polynomials $\rho_{J}$ are sums of these monomials, and coefficients of monomials

$$
1, \quad t_{3}, \quad t_{1} t_{3}, \quad t_{1} t_{3} t_{5}
$$


in $\rho_{J}$ are given in the following table.

\begin{tabular}{l|cccc} 
& 1 & $t_{3}$ & $t_{1} t_{3}$ & $t_{1} t_{3} t_{5}$ \\
\hline$\rho_{01|23| 45}=\left(1+t_{2}+t_{2} t_{3}\right)\left(1+t_{4}+t_{4} t_{5}\right)$ & 1 & 0 & 0 & 0 \\
$\rho_{01|24| 35}=\left(1+t_{2}+t_{2} t_{4}\right)\left(1+t_{3}+t_{3} t_{5}\right)$ & 1 & 1 & 0 & 0 \\
$\rho_{02|13| 45}=\left(1+t_{1}+t_{1} t_{3}\right)\left(1+t_{4}+t_{4} t_{5}\right)$ & 1 & 0 & 1 & 0 \\
$\rho_{02|14| 35}=\left(1+t_{1}+t_{1} t_{4}\right)\left(1+t_{3}+t_{3} t_{5}\right)$ & 1 & 1 & 1 & 1
\end{tabular}

This $4 \times 4$ matrix is unimodular. Hence $\oplus \mathbb{Z} \rho_{J}$ is primitive in $\oplus \mathbb{Z} t^{q}$ and (4.1) is free.

To compute the primitive sublattice $\mathcal{L}_{\mathcal{K}}$ explicitly, we note that

Lemma 4.2. Let $X$ be a smooth cubic fourfold, $S$ and $T$ be planes in $X$. Then we have

$$
[S] \cdot[T]= \begin{cases}0 & (S \cap T=\phi) \\ 1 & (S \cap T=a \text { point }) \\ -1 & \left(S \cap T=\mathbb{P}^{1}\right) \\ 3 & (S=T)\end{cases}
$$

Proof. The first and the second case are obvious. In the case of $S=T$, the self-intersection number $[S] \cdot[S]$ is given by the 2 nd Chern class $c_{2}\left(N_{S / X}\right)$ of the normal bundle $N_{S / X}$. If $S \cap T=\mathbb{P}^{1}$, the intersection number is given by the coefficient of $t$ in the power series

$$
\frac{c_{t}\left(N_{S / X}\right) c_{t}\left(N_{T / X}\right)}{c_{t}\left(N_{\mathbb{P}^{1} / X}\right)}
$$

From $c_{t}\left(N_{\mathbb{P}^{2} / X}\right)=1+3 t^{2}$ and $c_{t}\left(N_{\mathbb{P}^{1} / X}\right)=1+t$, we obtain intersection numbers.

Therefore compuations of intersection numbers $\left[L_{I, \alpha}\right] \cdot\left[L_{J, \beta}\right]$ are reduced to that of ranks of linear equations for $L_{I, \alpha} \cap L_{J, \beta}$. Using a computer, we can show that

Lemma 4.3. The sublattice $\mathcal{L}_{\mathcal{K}} \subset H^{4}\left(X_{0}, \mathbb{Z}\right)$ is of rank 19 , and its discriminant is 81 . Moreover we have $\left(\mathcal{L}_{\mathcal{K}}\right)^{\perp}=\operatorname{Im} \phi$, and hence $\operatorname{Im} \phi$ is a primitive sublattice of $H^{4}\left(X_{0}, \mathbb{Z}\right)$.

Proof. Let us consider 19 planes $S_{1}, \ldots, S_{19}$ in the following table.

\begin{tabular}{|c|c|c||c|c|c||c|c|c||c|c|c|}
\hline$L_{J, \beta}$ & $J$ & $\beta$ & $L_{J, \beta}$ & $J$ & $\beta$ & $L_{J, \beta}$ & $J$ & $\beta$ & $L_{J, \beta}$ & $J$ & $\beta$ \\
\hline$S_{1}$ & $J_{1}$ & $(1,1,1)$ & $S_{6}$ & $J_{1}$ & $(\omega, 1,1)$ & $S_{11}$ & $J_{2}$ & $(1,1, \omega)$ & $S_{16}$ & $J_{3}$ & $(1, \omega, 1)$ \\
$S_{2}$ & $J_{1}$ & $(1,1, \omega)$ & $S_{7}$ & $J_{1}$ & $(\omega, 1, \omega)$ & $S_{12}$ & $J_{2}$ & $(\omega, 1,1)$ & $S_{17}$ & $J_{3}$ & $(1, \omega, \omega)$ \\
$S_{3}$ & $J_{1}$ & $\left(1,1, \omega^{2}\right)$ & $S_{8}$ & $J_{1}$ & $(\omega, \omega, 1)$ & $S_{13}$ & $J_{2}$ & $(\omega, 1, \omega)$ & $S_{18}$ & $J_{4}$ & $(1,1,1)$ \\
$S_{4}$ & $J_{1}$ & $(1, \omega, 1)$ & $S_{9}$ & $J_{1}$ & $(\omega, \omega, \omega)$ & $S_{14}$ & $J_{3}$ & $(1,1,1)$ & $S_{19}$ & $J_{4}$ & $(1,1, \omega)$ \\
$S_{5}$ & $J_{1}$ & $(1, \omega, \omega)$ & $S_{10}$ & $J_{2}$ & $(1,1,1)$ & $S_{15}$ & $J_{3}$ & $(1,1, \omega)$ & & & \\
\hline
\end{tabular}

Using the previous Lemma, we can compute the intersection matrix $M$ of these 19 planes (see Appendix) and we have $\operatorname{det} M=81$. Since $\mathcal{L}_{\mathcal{K}}$ is orthogonal to $\operatorname{Im} \phi$ (which is of rank 4) in Lemma 3.2, we have

$$
\left(\left\langle\left[S_{1}\right], \ldots,\left[S_{19}\right]\right\rangle_{\mathbb{Z}} \oplus \operatorname{Im} \phi\right) \otimes \mathbb{Q}=H^{4}\left(X_{0}, \mathbb{Q}\right) .
$$

Therefore these 19 planes form a basis of $\mathcal{L}_{\mathcal{K}} \otimes \mathbb{Q}$. To complete the proof, we need to show that 108 planes $L_{J, \beta}$ are represented by $\mathbb{Z}$-linear combinations of $S_{1}, \ldots, S_{19}$ in $H^{4}\left(X_{0}, \mathbb{Z}\right)$. Using a computer, we can check this by the following way. For $J \in \mathcal{K}$ and $\beta \in \mathcal{B}$, let $M_{J, \beta}$ be the intersection matrix of 20 planes $S_{1}, \ldots, S_{19}, L_{J, \beta}$. We can check that $\operatorname{det} M_{J, \beta}=0$ and the eigenspace of 0 is 1 -dimensional. Moreover we have an integral eigenvector of the form

$$
\left(m_{1}, m_{2}, \ldots, m_{19}, 1\right) \in \mathbb{Z}^{20}
$$

and then

$$
m_{1}\left[S_{1}\right]+m_{2}\left[S_{2}\right]+\cdots+m_{19}\left[S_{19}\right]+\left[L_{J, \beta}\right]=0
$$

in $H^{4}\left(X_{0}, \mathbb{Z}\right)$, since it is orthogonal to $\left[S_{i}\right]$ and $\operatorname{Im} \phi$. 
Note that our cubic fourfolds $X$ are obtained as deformations of the Fermat cubic $X_{0}$, with diagram 3.1. Therefore $\operatorname{Im} \phi$ is primitive in $H^{4}(X, \mathbb{Z})$ for other $X$. Now Theorem 1.7 follows from facts that $\phi$ is a morphism of Hodge structures and $\phi_{\mathbb{C}}$ maps

$$
\left[H^{1,0}\left(C_{1}\right) \otimes H^{1,0}\left(C_{2}\right)\right] \oplus\left[H^{0,1}\left(C_{1}\right) \otimes H^{0,1}\left(C_{2}\right)\right]
$$

to $H^{3,1}(X) \oplus H^{1,3}(X)$, and $H^{1}\left(C_{1}, \mathbb{Q}\right) \otimes H^{1}\left(C_{2}, \mathbb{Q}\right)$ is indecomposable as a Hodge structure for general $C_{1}$ and $C_{2}$.

\section{ApPEndix A. Intersection MATRIX}

Let II be a $19 \times 19$ matrix all of whose entries are 1 . Let $M$ be the intersection matrix of $S_{1}, \ldots, S_{19}$ in the proof of Lemma 4.3. Then the matrix $M+\mathbb{I}$ (to avoid using the minus sign, we added I) is given by

$$
M+\mathbb{I}=\left(\begin{array}{lllll|lllll|lllll|llll}
4 & 0 & 0 & 0 & 2 & 0 & 2 & 2 & 1 & 0 & 2 & 2 & 1 & 0 & 2 & 2 & 1 & 2 & 1 \\
0 & 4 & 0 & 2 & 0 & 2 & 0 & 1 & 2 & 2 & 0 & 1 & 2 & 2 & 0 & 1 & 2 & 1 & 2 \\
0 & 0 & 4 & 2 & 2 & 2 & 2 & 1 & 1 & 2 & 2 & 1 & 1 & 2 & 2 & 1 & 1 & 1 & 1 \\
0 & 2 & 2 & 4 & 0 & 2 & 1 & 0 & 2 & 2 & 2 & 1 & 1 & 2 & 1 & 0 & 2 & 1 & 1 \\
2 & 0 & 2 & 0 & 4 & 1 & 2 & 2 & 0 & 0 & 2 & 2 & 1 & 1 & 2 & 2 & 0 & 2 & 1 \\
\hline 0 & 2 & 2 & 2 & 1 & 4 & 0 & 0 & 2 & 2 & 1 & 0 & 2 & 2 & 1 & 2 & 1 & 1 & 2 \\
2 & 0 & 2 & 1 & 2 & 0 & 4 & 2 & 0 & 1 & 2 & 2 & 0 & 1 & 2 & 1 & 2 & 1 & 1 \\
2 & 1 & 1 & 0 & 2 & 0 & 2 & 4 & 0 & 1 & 1 & 2 & 2 & 0 & 2 & 2 & 1 & 2 & 1 \\
1 & 2 & 1 & 2 & 0 & 2 & 0 & 0 & 4 & 2 & 1 & 0 & 2 & 2 & 0 & 1 & 2 & 1 & 2 \\
0 & 2 & 2 & 2 & 0 & 2 & 1 & 1 & 2 & 4 & 0 & 0 & 2 & 2 & 1 & 1 & 2 & 0 & 2 \\
\hline 2 & 0 & 2 & 2 & 2 & 1 & 2 & 1 & 1 & 0 & 4 & 2 & 0 & 1 & 2 & 1 & 1 & 2 & 0 \\
2 & 1 & 1 & 1 & 2 & 0 & 2 & 2 & 0 & 0 & 2 & 4 & 0 & 1 & 2 & 1 & 1 & 2 & 1 \\
1 & 2 & 1 & 1 & 1 & 2 & 0 & 2 & 2 & 2 & 0 & 0 & 4 & 1 & 1 & 2 & 1 & 1 & 2 \\
0 & 2 & 2 & 2 & 1 & 2 & 1 & 0 & 2 & 2 & 1 & 1 & 1 & 4 & 0 & 0 & 2 & 0 & 2 \\
2 & 0 & 2 & 1 & 2 & 1 & 2 & 2 & 0 & 1 & 2 & 2 & 1 & 0 & 4 & 2 & 0 & 2 & 0 \\
\hline 2 & 1 & 1 & 0 & 2 & 2 & 1 & 2 & 1 & 1 & 1 & 1 & 2 & 0 & 2 & 4 & 0 & 2 & 2 \\
1 & 2 & 1 & 2 & 0 & 1 & 2 & 1 & 2 & 2 & 1 & 1 & 1 & 2 & 0 & 0 & 4 & 0 & 2 \\
2 & 1 & 1 & 1 & 2 & 1 & 1 & 2 & 1 & 0 & 2 & 2 & 1 & 0 & 2 & 2 & 0 & 4 & 0 \\
1 & 2 & 1 & 1 & 1 & 2 & 1 & 1 & 2 & 2 & 0 & 1 & 2 & 2 & 0 & 2 & 2 & 0 & 4
\end{array}\right)
$$

\section{REFERENCES}

[Be14] A. Beauville, Some surfaces with maximal Picard number, J. Éc. polytech. Math. 1 (2014), 101-116.

[BS07] S. Boissiére and A. Sarti, Counting lines on surfaces, Ann. Sc. Norm. Super. Pisa, Cl. Sci. 6 (2007), 39-52.

[BS08] S. Boissière and A. Sarti, On the Néron-Severi group of surfaces with many lines, Proc. Amer. Math. Soc. 136 (2008), no. 11, 3861-3867.

[BM17] A. Bonifant and J. Milnor, On real and complex cubic curves, Enseign. Math. 63 (2017), no. 1-2, 21-61. [CHM95] L. Caporaso, J. Harris, and B. Mazur, How many rational points can a curve have?, The moduli space of curves, Progr. Math., vol. 129, Birkhaüser Boston, Boston, MA, 1995, 13-31.

[DIO21] A. Degtyarev, I. Itenberg and J. C. Ottem, Planes in cubic fourfolds, arXiv:2105.13951v1 [math.AG]

[DS16] A. Degtyarev and I. Shimada, On the topology of projective subspaces in complex Fermat varieties, J. Math. Soc. Japan 68 (2016), no. 3, 975-996.

[EH16] D. Eisenbud and J. Harris, 3264 and All That: A Second Course in Algebraic Geometry, Cambridge University Press (2016).

[H00] B. Hassett, Special cubic fourfolds, Compositio Mathematica 120 (2000), 1-23.

[RS15] S. Rams and M. Schutt, 64 lines on smooth quartic surfaces, Math. Ann. 362 (2015), 679-698.

[Se47] B. Segre, On arithmetical properties of quartic surfaces, Proc. London Math. Soc. (2) 49 (1947), 353-395.

[Sh79] T. Shioda, The Hodge conjecture for Fermat varieties, Math. Ann. 245 (1979), no. 2, 175-184.

[SK79] T. Shioda and T. Katsura, On Fermat varieties, Tohoku Math. J. (2) 31 (1979), no.1, 97-115.

[SM74] T. Shioda and N. Mitani, Singular abelian surfaces and binary quadratic forms, Classification of algebraic varieties and compact complexmanifolds, pp. 259-287. LNM 412 (1974), Springer, Berlin.

[V07] C. Voisin, Some aspects of the Hodge conjecture, Japan. J. Math. 2 (2007), 261-296. 